\title{
Proceedings of the joint summer meeting of the British and American Neuropsychiatry Associations, 12-14 July 1992, Oxford
}

The first joint meeting of the British and American Neuropsychiatry Associations was held at St. Catherine's College, Oxford in July. This was the second residential summer meeting for the British contingent and a chance to meet colleagues from the USA. The topics of the meeting were: paroxysmal disorders, head injury and the USA/UK views on "what is neuropsychiatry".

The paroxysmal disorder session encompassed a variety of topics such as epilepsy, migraine, panic disorder and transient global amnesia. Dr R Green (Atlanta) talked about the neuropathology of temporal lobe epilepsy. His contribution focused on the role of hippocampal sclerosis in temporal lobe epilepsy. This lesion, the commonest abnormality found in temporal lobes resected for control of epilepsy, is generally associated with good postsurgical epileptic control and is likely to follow rather than be the initial cause of epileptic activity. The neuronal loss and other cytoarchitectural abnormalities found in hippocampal sclerosis are prominent in some areas of Ammon's horn (CA1, CA3 and CA4) but spare others (CA2).

Animal studies suggest that hippocampal damage is mediated by excitatory amino acid neurotransmitters. Based on his own data $\mathrm{Dr}$ Green described sprouting of mossy fibres into the molecular layer of the dentate gyrus and suggested that this aberrant growth may create a substratum for recurrent excitation leading to further epileptic activity. These anatomical changes are accompanied by an increase in kainic and NMDA receptor binding, suggesting a mediating role for excitatory amino acids. These limbic and hippocampal abnormalities are likely to have behavioural counterparts, but the precise link between the two remains elusive.

The impact of epileptic activity on the nervous system was discussed by Dr E Reynolds (London) from a clinical perspective. Large follow up studies have shown that $70-80 \%$ of newly diagnosed patients with epilepsy experience a two to five year remission, and that only a quarter enter an early chronic course. In his own series of over 400 newly diagnosed patients, persistence of seizures a year later was related to the number of seizures the patient had before the onset of treatment. Seizure frequency and type of anticonvulsant used were of little prognostic value. These observations are in keeping with the possibility that structural and biochemical abnormalities resulting from epileptic seizures are in turn epileptogenic. In his view the argument for treating single seizures is gaining momentum.

Dr J Bird (Bristol) dealt with the role of psychological factors in the precipitation and inhibition of seizures. In his own series, 92 of 100 epileptic patients associated increased seizure frequency with at least one psychological factor. Feeling anxious, premenstrual tension and feeling depressed were the most commonly endorsed precipitants. A feeling of happiness and relaxation was associated by many with a decrease in seizure frequency. Patients with poorly controlled epilepsy and those receiving several anticonvulsants were more likely to report these associations. Fifteen per cent believed that they could abort seizures and a similar proportion, often the same patients, that they could precipitate them. These findings may have some therapeutic implications in treatment resistant epilepsy. The complex interaction of environmental, personality and organic factors in determining abnormal paroxysmal behaviour was further discussed by Dr P Taylor (London) in her talk on the neuropsychiatry of pathological violence.

Dr F Clifford Rose (London) discussed current views on the pathogenesis of migraine and the evidence suggesting that even between attacks the brain of the migraine sufferer is not "normal". A novel approach to the pathogenesis and treatment of headaches and other paroxysmal disorders was put forward by Dr A Wilkins (Cambridge) who presented data suggesting that commonly encountered visual stimulation, such as that provided by some types of gas-discharge lighting or closely spaced text, may contribute to the causation of headaches, seizures and anxiety, and that coloured ophthalmic lenses may reduce these adverse effects.

Dr D M Clark (Oxford) expanded on the cognitive theory of panic, which views the disorder as resulting from an erroneous, catastrophic interpretation of a wide variety of bodily sensations. Several predictions arising from this theory have been tested by Dr Clark and colleagues, namely that those with panic attacks are more likely to use catastrophic interpretations than normal controls, that manipulation of these interpretations will precipitate or control panic attacks, and that the long term outcome of treatment will depend on whether cognitive change takes place. Of considerable interest is the effect that cognitive therapy was observed to have in preventing panic attacks in a group of patients who received lactate infusions. In the treatment trials carried out by Dr Clark, $50 \%$ of those treated with imipramine or relaxation were free 
from attacks a year later and that was so for $80 \%$ of those who received cognitive therapy. Only $7 \%$ of those who did not receive treatment improved spontaneously.

Anxiety disorder in patients with Parkinson's disease (PD) was reviewed by Drs R Schiffer and E Szigethy (Rochester, USA). Their phenomenological studies suggest that anxiety is a common feature of the affective disorders often encountered in Parkinson's disease (half of the patients in their series), a much higher prevalence than that found in depressed patients with MS. In a small group of PD patients with panic disorder, attacks were precipitated by the administration of yohimbine, an alpha- 2 antagonist, as it is the case in younger subjects without neurological disease, suggesting that the mechanism of panic attacks is common to both.

Dr J Hodges (Cambridge) presented the results of his study of transient global amnesia (TGA) with special emphasis on its aetiology. There was very little evidence coming from his study of over 100 patients to support a vascular aetiology. TGA patients when compared with those with transient ischaemic attacks (TIA) had fewer vascular risk factors and did not experience comparable rates of vascular events at follow up. TGA patients had migraine more often than a normal control population and 8 of 114 developed epilepsy within a year of their TGA. However, epilepsy is unlikely to be an aetiological factor in more than a minority of cases. In those in whom neurological signs were present and who therefore did not meet strict inclusion criteria, thrombo-embolic disease was likely to have been relevant. Inclusion of this group of patients in previous studies is likely to account for the reported discrepancies in aetiology and prognosis.

The session on head injury was open by $\mathrm{Dr}$ $M$ Trimble (London) who dealt with medicolegal aspects, giving invaluable practical advice to those who ever have to write a medico-legal report. Dr E Coffey (Pittsburg) presented the results of his follow up study of traumatic brain injury in the elderly. In his series of over 2000 patients poor outcome (defined in terms of mortality, length of hospital stay and degree of nursing care required after discharge) was related to increasing age. In addition, within the elderly population structural brain changes antedating the injury (for example, ventricular dilatation detected by CT) also carried a poor prognosis.

Professor Meldrum (London), discussed the role of excitotoxicity in traumatic brain injury, suggesting that after the early increase in extracellular glutamate concentration, ischaemia, caused by raised intracranial pressure, may lead to excitotoxic neuronal damage. Other mechanisms such as the action of toxins produced by microglia and macrophages on NMDA receptors may also be relevant. The fact that hippocampal damage is a frequent finding at postmortem, even in those who had not experienced post-traumatic anoxia, supports the role of excitotoxicity. This research opens up a whole range of therapeutic interventions which may become common place in the future. These may include the use of agents to reduce ischaemia-induced glutamate release (for example, lamotrigine), competitive and non-competitive NMDA receptor inhibitors and free radical scavengers.

Dr W McKinlay (Edinburgh) commented on the disabling psychosocial damage following severe head trauma. He discussed the benefits of neuropsychological rehabilitation, and concluded that memory aids such as check lists and diaries are often useful. Memory retraining requires the active participation of carers, and the success achieved in the target areas does not generalise to other problems. He regarded cognitive therapy as the most successful approach to deal with problems of temper control.

With a transcultural dimension in mind, Professor W A Lishman (London) gave his view on what is neuropsychiatry and $\operatorname{Dr} M$ Folstein (Baltimore), illustrated the USA approach by his discussion of research into Alzheimer's disease. Professor Lishman's lecture, the subject of an editorial in this issue of the journal, traced the development of the subspeciality in this country and contrasted USA/UK views as to its scope, drawing on the results of a questionnaire previously circulated to the members of both Associations. Whilst neuropsychiatry was widely regarded as a clinical approach to psychiatric illness, rather than restricted to the study of a limited number of conditions, opinions were more divided on the standing of neuropsychiatry in relation to biological psychiatry.

A significant majority of UK members felt that neuropsychiatry could be included within the field of biological psychiatry, whilst more USA members favoured the opposite view. A difference between neurologists and psychiatrists was also apparent, with neurologists from both countries favouring the ascendancy of neuropsychiatry over biological psychiatry. A sharp distinction was drawn between neuropsychiatry and behavioural neurology by USA and UK respondents. Behavioural neurology, essentially an American subspeciality originating from neurology rather than psychiatry, focuses on defined areas of abnormal behaviour rather than on broad segments of mental disorder and is therefore more limited in its scope.

Dr Maria Wyke talked about the relevance of neuropsychology to neuropsychiatry. As in neurology, neuropsychological tests applied to psychiatric patients can give useful information about the strengths and weaknesses in cognitive functioning, provide baselines for future reference and set goals for rehabilitation. Dr Wyke, however, emphasised the hazards involved in translating the "anatomical" diagnostic model, often appropriate in neurology, to psychiatry problems. She made a plea for careful interpretation of test results in psychiatric patients, and expressed a hope for the development of a new brand of neuropsychology with roots firmly established in neuropsychiatry itself rather than based on the more traditional lesional model.

It is now customary in these meetings to 
have a session dedicated to clinical presentations and this time there was the added interest of having the chairman and commentators from the opposite side of the Atlantic. This produced a lively discussion of the cases presented, which brought to light the similarities rather than the differences in clinical practice between the UK and USA. Those presenting cases were Drs S Salloway and J
Duffy (USA) and Drs M Kopelman, D Jadresic, $S$ Lewis, $\mathrm{K}$ Barrett and $\mathrm{M}$ Lambert (UK).

The success of this first joint meeting led to tentative proposals for similar ventures in the future. In the meantime, the winter meeting will take place in London on 22 January 1993 and the subject will be cognitive abnormalities in neuropsychiatric disorders.

\section{Domenico Cotugno CSF, and the origins of sci- atica}

Near the heel of Italy lies the town of Ruvo di Puglia, the birthplace of Domenico Cotugno (1736-1822) Educated in the world's oldest school of medicine at Salerno, Cotugno spent most of his life working in Naples where he became the Professor of anatomy. He is best known for his work on CSF "Liquor Cotugnii" which despite the publications of "Swedenborg the Obscure", and Vieussens' description of clear ventricular fluid was not generally understood. At a time when nerve juice-succus nerveus had just replaced Galen's $\pi v \varepsilon v \mu a$ $\psi v \chi$ lkov.

To prove the free circulation between the cranial and spinal dura, Cotugno stood cadavers on their feet and decapitated them to observe the flow of CSF. He described hydrocephalus ex vacuo. He also noted that $\mathrm{CSF}$ was incoagulable in health but, like urine, where there was disease became cloudy on boiling. "The cerebrospinal water is in perenni statu renovationis, through exudation by minimal arteries and reabsorption by minimal veins. It penetrates into dural sleeves of nerve roots; hence is apt to accumulate in the sheaths of the sciatic nerve and so give pain along its course. Such pain, weakness, and limping may be cured; if necessary by vesicants and caustics to draw out the hydrops".'2
JMS PEARCE 1 Schiller F. Domenico Cotugno. In: Webb Haymarker,
Francis Schiller, eds. The founders of neurology, 2nd ed. Springfield. Charles C Thomas, 1970:19-23.

2 Cotugno D. De ischiade nervosa commentarius. Naples: Simoni, 1764:9-14 (cited by Schiller F). 